\title{
A method for locating rockfall impacts using signals recorded by a microseismic network

\author{
Teresa Gracchi ${ }^{1^{*}}$ D, Alessia Lotti ${ }^{1}$, Gilberto Saccorotti ${ }^{2}$, Luca Lombardi ${ }^{1}$, Massimiliano Nocentini ${ }^{1}$, \\ Francesco Mugnai ${ }^{1,3}$, Giovanni Gigli ${ }^{1}$, Marco Barla ${ }^{4}$, Andrea Giorgetti ${ }^{5}$, Francesco Antolini ${ }^{4}$, Andrea Fiaschi ${ }^{6}$, \\ Luca Matassoni ${ }^{6}$ \\ and Nicola Casagli ${ }^{1}$
}

\begin{abstract}
Background: Rockfall events are one of the most dangerous phenomena that often cause several damages both to people and facilities. During recent years, the scientific community focused the attention at evaluating the effectiveness of seismological methods in monitoring these phenomena. In this work, we present a quick and practical method to locate the rebounds of some man-induced boulders falls from a landslides crown located in the Northern Apennines (Central Italy). The reconstruction of the trajectories was obtained by means of back analysis performed through a Matlab code that takes into account both the DEM (Digital Elevation Model) of the ground, the geotechnical-geophysical characteristics of the slope and the arrival times of the seismic signals generated by the rock impacts on the ground.

Results: The localization results have been compared with GPS coordinates of the points and videos footage acquired during the simulations, in order to assess the reliability of the method. In most cases, the retrieved impact points match with the real trajectories, showing a high reliability. Furthermore, four different cases have been identified as a function of the geomechanical, geophysical and morphological conditions. Due to the latter ones, in some case it was necessary to assume different values for the propagation velocity of the elastic waves in the ground, here assumed to be isotropic and homogeneous.

Conclusions: This work aims at evaluating the effectiveness of a quick and practical method to locate rockfall events using a small-aperture seismic network. The obtained results indicate that the technique can provide quantitative information about the area most prone to impact of detached blocks. The method still presents some uncertainty, but reducing some of the approximations (e.g. by better constraining the velocity model), it could lead to prompt and more accurate results, easily applicable to hazard estimates.
\end{abstract}

Keywords: Seismic monitoring, Seismic network, Rockfall simulation, Rockslide, Localization, Traveltime function

\section{Background}

Landslides are frequent and widespread geomorphological phenomena that often cause huge damages. Italy is one of the country more prone to landslides (Classified European Landslide Susceptibility Map, Günther et al. 2014). One of the greatest risk factor is the occurrence of boulders detachments from unstable rock slopes, potentially dangerous for people

\footnotetext{
* Correspondence: teresa.gracchi@unifi.it

${ }^{1}$ Department of Earth Sciences, University of Florence, Via Giorgio La Pira 4, 50121 Florence, Italy

Full list of author information is available at the end of the article
}

and goods. During last years news often highlighted the spread of these potentially lethal events (10/5/ 2010 Val Trebbia, Piacenza; 9/8/2011 Trentino). Therefore, it is necessary to gain further insights on these phenomena, with the aim to better comprehend the behavior of a rock falling down from a slope, to subsequently identify the areas where such phenomena could occur.

In particular, the identification of areas where a rockfall might happen, allows for the implementation of stabilization or protective measures before the occurrence of catastrophic events (Baillifard et al. 2003). The analysis 
of rockfall trajectories in fact, is essential to calibrate, design and distribute mitigation measures. Moreover, this turns into significant saving of money, according to Shuster and Leighton (1988) who estimated that if appropriate strategies are adopted, at least the losses caused by instability phenomena can be reduced by more than $90 \%$.

In addition to the most common application of seismology (Allen, 1978; Jongmans and Garambois, 2007) as prospecting, sliding surface identification (Bruno \& Martillier, 2000; McCann \& Forster, 1990) and microzonation (Bour et al., 1998; Scott et al., 2006), seismic and microseismic networks have already been applied to study rockslide (Helmstetter and Garambois, 2010; Deparis et al. 2008; Dammeier et al. 2011), showing that seismic signals can provide interesting information on rockfall events (Norris, 1994; Dammeier et al., 2016; Hibert et al., 2014; Lacroix and Helmstetter, 2011). Indeed, crack propagation generate micro-seismic events (Manconi et al. 2016).

Furthermore, seismic measurements could be suitable for this purpose since they are non-invasive methods and are relatively inexpensive (Vilajosana et al. 2008).Within this framework, numerous researchers focused on the localization of rockfall events, with the aim to implement the proposed technique as a part of an early-warning system, able to quickly identify the most active portions of an unstable slope.

For this target, different techniques have been proposed in literature. In most cases, the localization of the impact points has been done using the polarization analysis of the seismic signal (Vilajosana et al. 2008; Levy et al. 2011); others (Bottelin et al. 2014; Lacroix and Helmstetter 2011) proposed the triangulation technique, or the beamforming method.

This study aims to localize rock impacts on the ground using the arrival times extracted from the traces with manual picking. This method is rarely used due to its complexity and the result loss of time (Dammeier et al. 2011; Colombero et al. 2016; Chen and Holland, 2016), especially when traces are affected by ambient noise. On the other hand, where a large number of data are not involved, the manual procedure can be a good compromise since it bars false misrepresents and allow some considerations concerning seismic noise and signal amplitude. Once the manual picking procedure has been carried out, the proposed method results to be very quick and practical, allowing localization without the need to study waveform, frequency content or amplitude, that can be required in other methods (e.g. the polarization analysis).

The work shows the accuracy of the method applied in our case study comparing the obtained results to the real blocks trajectories filmed during a field campaign when some man-induced rockfalls were carried on.

To reach the targets, we localized blocks impacts on the ground recorded as a single transient by the seismic network. The first step consisted in the manual picking of the arrival times, the second one focused on the localization of each detected transient to reconstruct the whole trajectory from the throw point to the arrival one (both measured also by GPS technique).

In some studies artificial rockfall events have been filmed in order to evaluate some parameters as velocity and energy (Berger et al. 2002; Vilajosana et al. 2008). In particular, a work similar to the one we are reporting, was carried on by Bottelin et al. (2014) during an event on the French Alps. In that case a rockslide was artificially triggered by blasting an unstable rock mass and a seismic network, recording in continuous, was settled. During the experiment, some cameras filmed the boulders and videos have been used to estimate the rockfalls velocities but not as a tool to verify the localization process reliability.

\section{Study area}

The chosen test site was located in a dismissed limestone quarry near the town of Assisi in the Northern Apennines, Central Italy (Fig. 1). The unstable wedge consists of a huge portion of the northwestward slope of Mount Subasio and involves a volume of $\sim 182.000 \mathrm{~m}^{3}$ (Intrieri et al. 2012). The collapse could threat county (S.P. 249) and state (S.S. 444) roads placed downstream the unstable wedge, which are the only connection between the town of Assisi and the villages in the surroundings (green tracks, Fig. 1).

The slope, oriented approximately along the SE-NW direction (with a dip of about $30-38^{\circ}$ ) is mainly made of Maiolica Formation which consists of well stratified micritic limestone (from $10 \mathrm{~cm}$ to $1 \mathrm{~m}$ ) with intercalation of thin clay layers. The rockslide is in the upper part of the quarry and has a rough trapezoidal shape. The upper boundary is associated to a big tension crack up to one meter wide and $100 \mathrm{~m}$ long, the lower one matches with the sliding surface that transversally cut the slope, and the western lateral boundary consists of a persistent fractures system derived from the coalescence of several joint families. The slide generally showed slow movements after heavy rainfalls (Ponziani et al. 2011, Intrieri et al. 2012), and due to the intense fracturing, it is particularly subject to rockfall phenomena.

Two major events, happened in 2004 and in 2005, and respectively involved a few tens of $\mathrm{m}^{3}$ and $2500 \mathrm{~m}^{3}$ (Graziani et al. 2009), getting the attention of the scientific community. Since then many studies have been carried out on the area by Alta Scuola di Perugia and the Department of Earth Sciences of the University of 


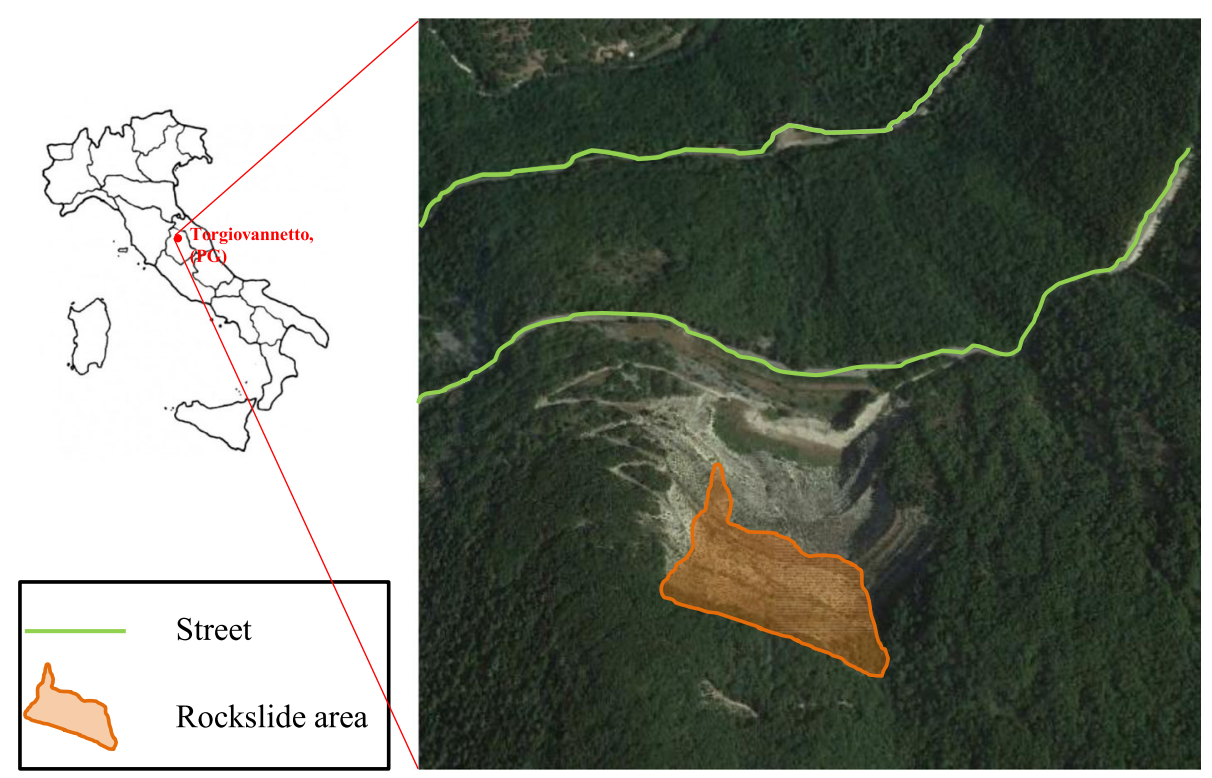

Fig. 1 The former limestone quarry of Torgiovannetto, Assisi (PG), Italy

Florence trying to identify some parameters able to forecast the landslide movements.

The rockslide (Cruden and Varnes, 1996) has been instrumented starting from summer 2007 with a network equipped with 13 wire extensometers, 1 thermometer, 1 rain gauge and 3 cameras (Intrieri et al. 2012) that continuously monitored the slope. A seismic network was installed on December 2012 (Lotti et al. 2015; Amorese et al. 2015) to supplement the monitoring system. Moreover a WSN system was installed in 2013 composed of 15 wireless nodes, where one of these acts as network coordinator (NC), 3 clinometers (tiltmeters), 4 wire extensometers, 2 bar extensometers, and 4 soil hygrometers in the framework of a National Research Project (PRIN 2009) in cooperation by University of Florence, University of Bologna and Politecnico di Torino (Giorgetti et al. 2016). A ground based radar interferometry campaign was also conducted as part of the project (Barla \& Antolini 2016, Antolini et al. 2016).

\section{Field survey and instrumentation}

For the above mentioned purposes, the Department of Earth Sciences of the University of Florence tested the application of a microseismic network. The network,

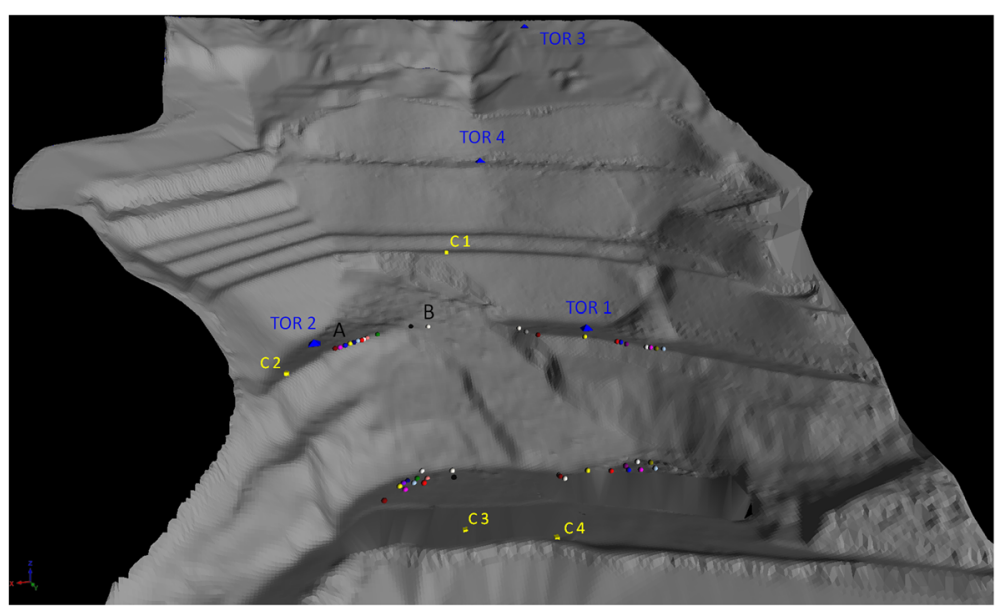

Fig. 2 Location of the seismic stations (blue pyramids) on Digital Terrain Model (DTM) of Torgiovannetto quarry. Some launch and arrival points are reported on the DTM as dots. All the tracks were filmed by the four cameras positioned at different heights (yellow cubes). C1, C2, C3 and C4 represents the Canon 660D, Nikon D700 and the two Canon EOS 600D cameras respectively. A and B represents two launch areas to which correspond different fall behavior (as explained in 'results and discussions' section) 


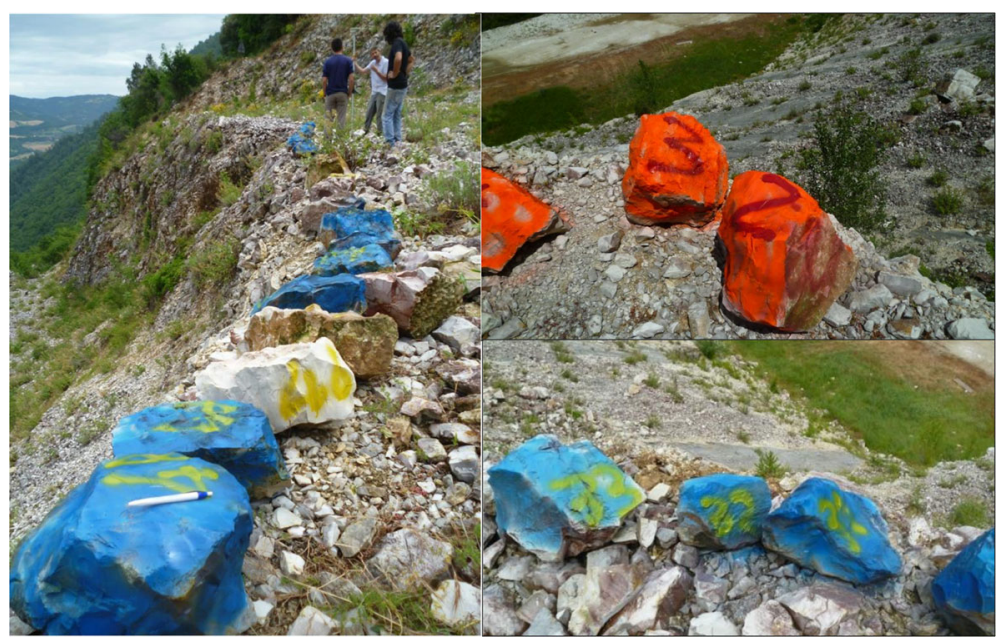

Fig. 3 Some of the blocks painted in bright colors and numbered, used for the man-induced rockfalls simulation

equipped with four stations, has been installed in collaboration with the Parsec Foundation (former Prato Ricerche). The stations acquired data in continuous mode from December 2012 to July 2013.

Figure 2 shows the location of each station: three outside the landslide body (TOR1, TOR2, TOR3) and one (TOR4) over it. Each station consists of a S45 tri-axial seismometer with a natural frequency of $4.5 \mathrm{~Hz}$ cable-connected to a 24bit digitizer. SARA Electronic Instrument provided both the devices. The sampling frequency was set to $200 \mathrm{~Hz}$.
Data were recorded in miniSEED format (Halbert et al. 1988 ) and subsequently converted in SAC (Seismological Analysis Code, Goldstein et al., 2003; Goldstein \& Snoke, 2005) format for processing operations.

To calibrate and verify the performance of the applied techniques, we carried out two days of field campaign (25/06/2013 and 04/07/2013), during which we triggered some man-induced rockfalls hurling about 95 blocks from different points of the slope (some of them are reported as dots in Fig. 2).

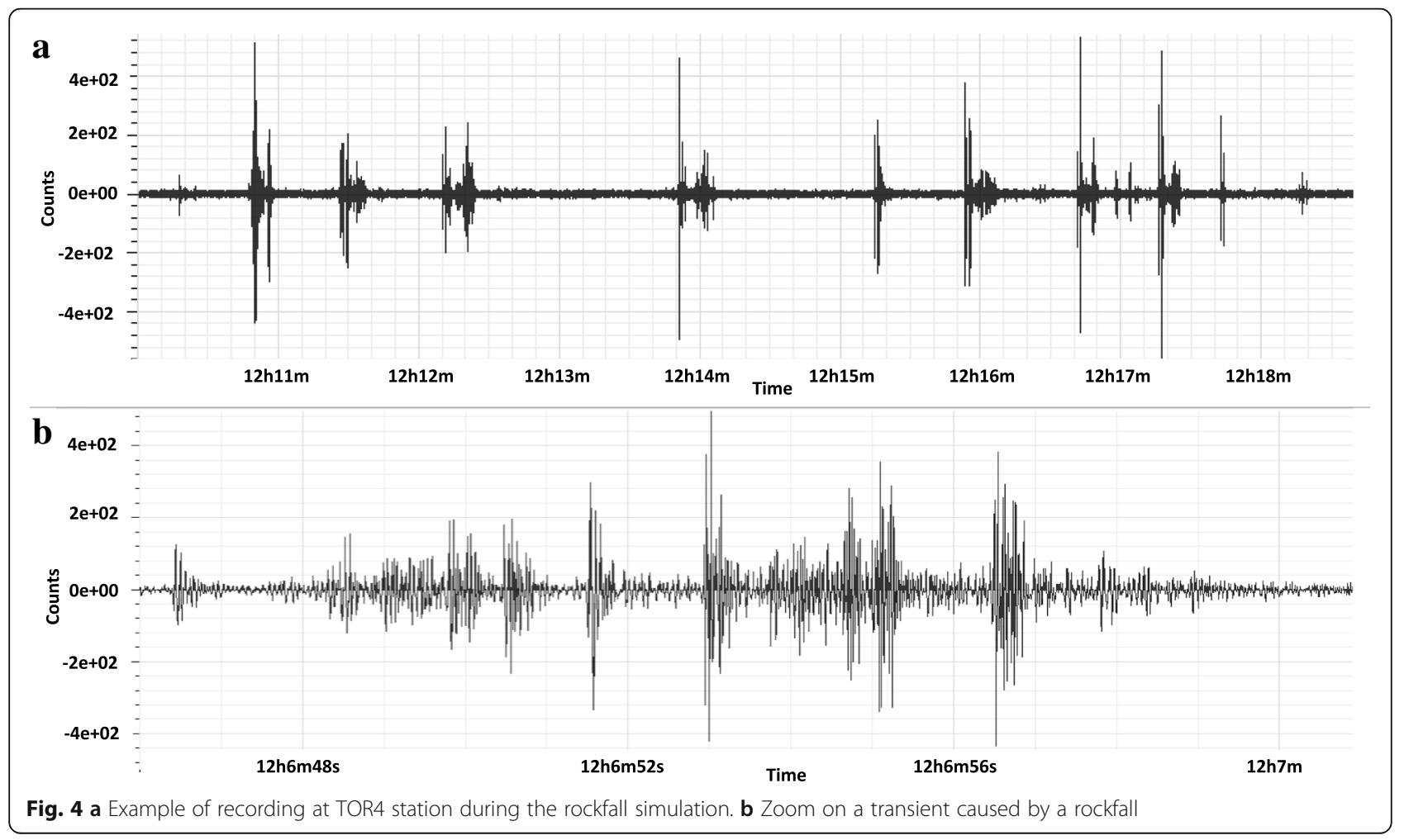




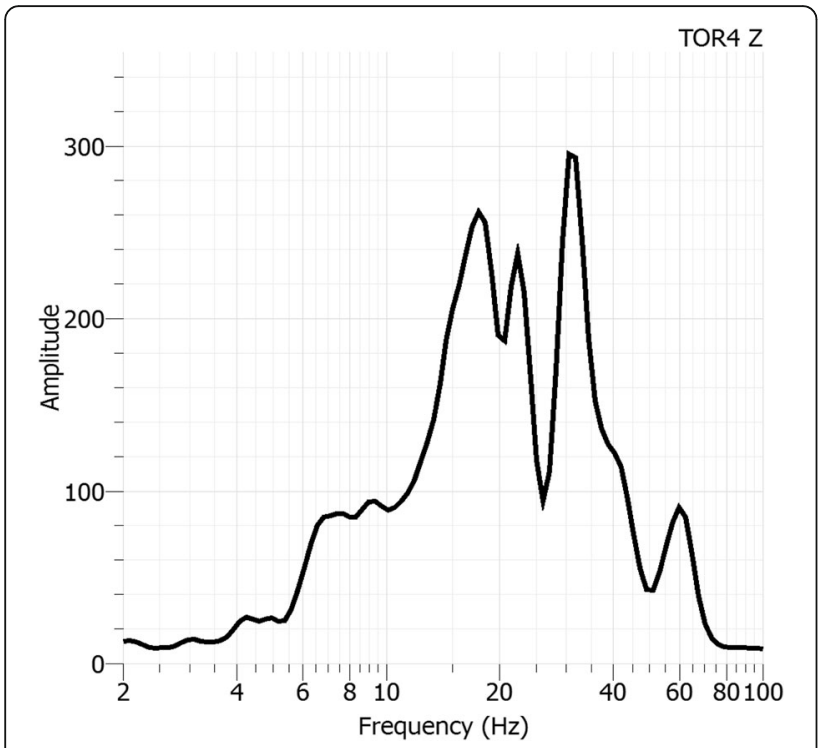

Fig. 5 Spectrum of the transient showed in Figure $4 \mathbf{b}$

During the entire experiment, each throw was filmed by four high resolution cameras (two Canon EOS 600D, one Canon 660D and a Nikon D700, yellow cubes, Fig. 2), to compare the impact locations as retrieved from analysis of the seismic traces with the effective trajectories of the falling blocks.

We also measured the coordinates of start and final (when possible) blocks' impact points with a dynamic GPS. Points coordinates were tracked on a georeferenced Digital Terrain Model (DTM). The DTM has been obtained from a point cloud acquired by a laser scanning survey carried out in July 2007.

The thrown rock blocks (ranging in size from $21 \times$ $20 \times 19 \mathrm{~cm}$ to $105 \times 58 \times 39 \mathrm{~cm}$ ) have been painted with bright colors in order to be better recognized in the videos, and numbered to be precisely related to their GPS coordinates (Fig. 3).

\section{Methods}

\section{Localization procedure}

The localization of the boulders' impact points was attained using an algorithm based on the non-linear inversion of seismic waves arrival times.

First, the slope has been parameterized by a regular grid of equally-spaced, geo-referenced nodes. The location procedure thus consists in an exhaustive grid search for those nodes at which the misfit function between measured and theoretical travel times is minimized. The localization problem has two unknowns: a) the hypocenter coordinates $X_{0}=\left(x_{0}, y_{0}, z_{0}\right)$ and $\left.\mathrm{b}\right)$ the origin time $t_{0}$. For a seismic network of $\mathrm{N}$ stations, the arrival times $t_{i}(i=1$... $\mathrm{N})$ at the generic $\mathrm{i}$-th station whose coordinates are given by the position vector $X_{i}=\left(x_{i}, y_{i}, z_{i}\right)$ are expressed as:

$$
t_{i}=t_{0}+\Delta\left(\dot{X}_{0}, ; \dot{X}_{i}\right)+e_{j}
$$

where $\Delta\left(\dot{X}_{0}, ; \dot{X}_{i}\right)$ is the travel time function and $e_{j}$ is the error due to the inaccuracy of the arrival time estimation and to the travel time function uncertainty.

Arrival times at individual stations are estimated through a manual picking procedure. At each grid node, the predicted travel times to the different stations are calculated under the assumption of isotropic and homogeneous medium (see later in this Section). Both sides of eq. 1 are then subtracted by their respective mean values calculated over the different stations of the network. This allows eliminating the origin time $t_{0}$ from the set of unknowns; eq. 1 is thus rewritten as:

$$
t_{i}-\dot{T}=\Delta\left(\dot{X}_{0}, ; \dot{X}_{i}\right)-\dot{\Delta}
$$

where $\dot{T}$ and $\dot{\Delta}$ are the station-averaged arrival times and travel times, respectively (Tarantola and Valette, 1982; Moser et al., 1992).

The theoretical travel times between each station and all the nodes of the gridded topographic surface (DTM) are then calculated assuming a homogeneous and isotropic medium:

$$
\left.\Delta \dot{X}_{0}, ; \dot{X}_{i}\right)=\frac{\sqrt{\left(x_{i}-x_{o}\right)^{2}+\left(y_{i}-y_{o}\right)^{2}+\left(z_{i}-z_{o}\right)^{2}}}{v}
$$

where $i$ is a generic station and $v$ is the constant propagation velocity assumed equal to $2000 \mathrm{~m} / \mathrm{s}$. The velocity has been chosen after several tests modifying the value from $1500 \mathrm{~m} / \mathrm{s}$ to $5000 \mathrm{~m} / \mathrm{s}$ that are typical values for the Maiolica formation (Rocca, 1983), with $500 \mathrm{~m} / \mathrm{s}$ as step. Comparing the results with the videos, $2000 \mathrm{~m} / \mathrm{s}$ was proved to be the most reliable velocity.

Finally, for any generic trial source located at X0, a misfit function is calculated through the $L^{2}$-norm of the difference between the measured times and the theoretical ones:

$$
\mathrm{R}(\mathbf{X 0})=\sum_{i=1}^{n s t a}\left|\left[t_{i}-\grave{T}\right]-\left[\Delta_{i}^{\text {theo }}-\dot{\Delta}\right]\right|^{2}
$$

Finally, the probability of location is expressed as:

$$
\mathrm{P}(\mathbf{X 0})=\exp \left(-0.5 \mathrm{R}(\mathbf{X 0}) / \sigma^{2}\right)
$$

where $\sigma^{2}$ is an error term which includes the uncertainties related to both picking errors and inaccuracies in travel time predictions due to incomplete knowledge of the velocity structure.

The solution is assumed to be associated with the grid node at which $\mathrm{P}(\mathbf{X 0})$ takes a maximum.

Values of $\mathrm{P}(\mathbf{X 0})$ are displayed as a colored surface superimposed on the gridded topographic surface; for each separate inversion, the grid node at which $\mathrm{P}(\mathbf{X 0})$ takes its maximum represent the most likely epicentral 


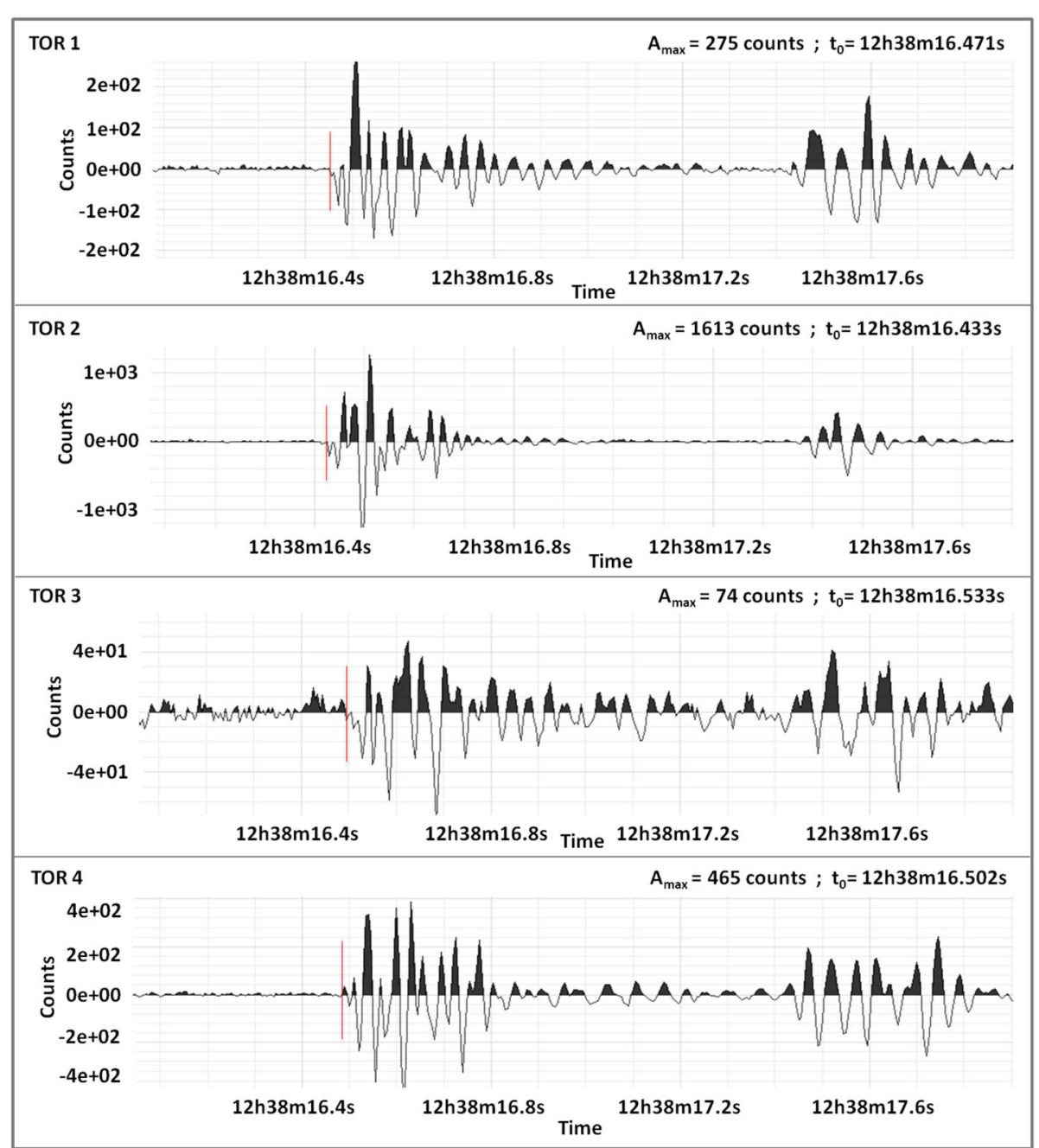

Fig. 6 Manual picking of the first impact's arrival time (highlighted in red) at stations TOR1, TOR2, TOR3 and TOR4, respectively from the top to the bottom. As an example, here is reported an event occurred at 12:38 UTC on 25th June 2013, first day of simulation. For each station, the maximum amplitude expressed in counts and the detected arrival time are pointed out. The signals are presented with different amplitude scales for visualization purposes

location (Saccorotti et al., 1998). When the energy of single consecutive rock impacts is high enough to generate well-distinguishable seismic pulses having clear onsets, then the location procedure is iterated to track, through the subsequent locations, the trajectories of the falling blocks.

\section{Seismic traces and manual picking procedure}

An example of some man-induced rockfall events recorded at the four stations is presented in Fig. 4. Traces have been displayed using GEOPSY (GEOPhisical Signal database for noise arraY processing, ver. 2.9.0). Figure 4a) point out the interval time from 12:10:00 to 12:20:00 UTC of June 25th, 2013 (day of simulation) recorded by TOR4 station. A rockfall event is generally recorded as a series of short impulses, whose time length spans from $3 \mathrm{~s}$ to $10 \mathrm{~s}$ while the number of rebounds were strictly influenced by the local topography (Fig. 4b). The involved frequencies are higher than those of the background noise or of other signals of different origin (e.g., earthquakes; Suriñach et al. 2005), in our case ranging from $10 \mathrm{~Hz}$ to $40 \mathrm{~Hz}$, with main peaks at $17 \mathrm{~Hz}$ and $30 \mathrm{~Hz}$ (Fig. 5).

Manual picking of the arrival time at each station has been done on the traces visualized in GEOPSY, by first extracting from the continuous seismic recordings only those portion encompassing artificial rockfalls. An example of manual picking of the arrival times at individual stations is shown in Fig. 6. Even if the microseismic network covers a small area $(200 \mathrm{~m} \times 100 \mathrm{~m})$, the signal's amplitude at the four stations changes significantly, as a consequence of the diverse station-to-source distances, which implies different geometrical spreading and inelastic attenuation. 
For the analysis that follow a MATLAB code was used. The analysis was carried out for the vertical component (SHZ) since it showed the best signal to noise ratio.

For both simulation days, traces recorded by TOR 3 resulted to be more difficult to analyze and pick because of the higher distance from the impact points (sources), that caused the signal attenuation. Moreover, TOR3 shows a lower signal-to-noise-ratio that can be explained considering that this station is located in the upper part of the quarry, surrounded by trees that, especially during windy days, induce vibrations to the ground concealing transient signals.

\section{Results and discussion}

The described procedure was applied to achieve the localization of subsequent impacts recorded during 57 rockfall simulations extracted from a database of 95 throws. Some of the throws have been discarded since the falls failed (the blocks immediately stopped) or the energy involved was too low and did not produce an event large enough to be detected by all stations.

The result of the localization procedure was the probability point cloud with its maximum value marked with green circles (Fig. 7b). In the image, an example showing an event occurred on 25th June 2013 at 12:06 (UTC) is reported, concerning a rock mass of dimension $23 \times 50 \times 23 \mathrm{~cm}$ (Fig. 7a). In that case, 4 impacts have been picked on the traces, denoting 4 rebounds (orange arrows, Fig. 7c). Table 1 shows the results of the manual picking, namely labels required for the elaboration process. For each one, the localization procedure has been applied to spatially identify the impact points and consequently to retrace the trajectory followed by the rock block. The red line shows the trajectory previously measured by GPS and videos analysis. In particular, the path was obtained by jointing the launch and arrival GPS points coordinates. Videos have been used to assure the reliability of this method, discarding too irregular trajectories. The probability map is relative to the last impact detected. As shown by Fig. 7b, green circles are aligned and quite close to the reconstructed trajectory.

As expected, the results of the boulders fall simulations were not homogenous: the launch position, and the eventual breakage of the blocks determined differences in the trajectories and the kinematic of the failed mass. This is clearly visible in the localization results.

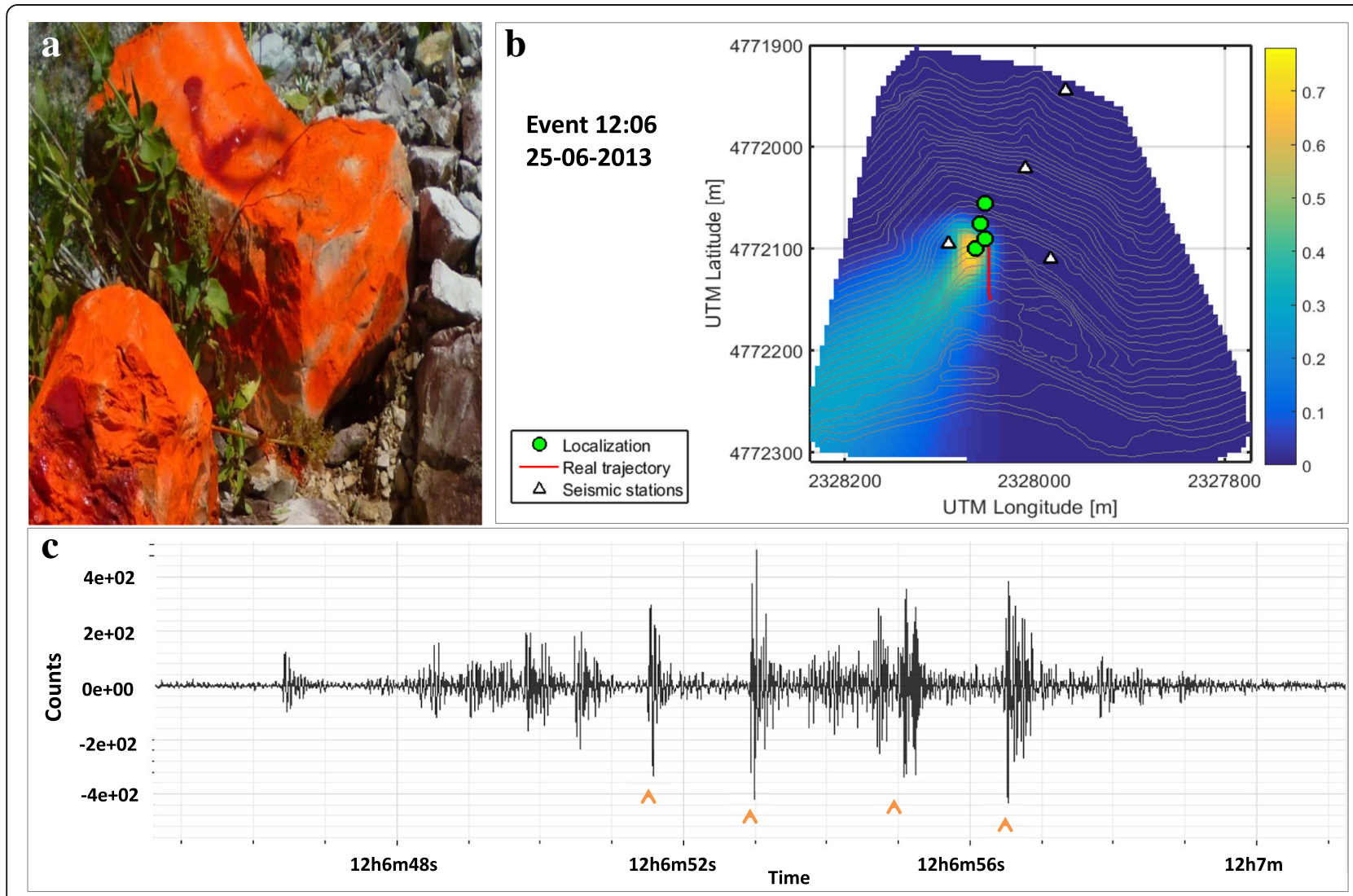

Fig. 7 Example of the localization procedure. a Block used for the simulation. b Localization output. Warm and cold bar colors indicate respectively high and low probability of the last impact. $\mathbf{c}$ Recording at TOR4 station. The orange arrows highlight peaks considered for the elaboration process 
Table 1 Arrival times at TOR4 station obtained by manual picking. Event occurred on 25th June 2013 at 12:06 UTC

\begin{tabular}{lllll}
\hline & Impact 1 & Impact 2 & Impact 3 & Impact 4 \\
\hline TOR4 & 120651.485 & 120652.924 & 120654.983 & 120656.454 \\
\hline
\end{tabular}

In particular, three different cases have been identified:

\section{CASE1: Clear rebounds}

This case concerns all the signals generated by blocks launched approximately from steps 1 to $5 \mathrm{~m}$ high. They rebound on the slope until they stopped, as it was also observed in both seismic recordings and video footages. The recorded signals were characterized by a sequence of short-duration transients, each one usually shorter than $1 \mathrm{~s}$ (Fig. 8c, d).

Figure 8 illustrates the results of two experiments held on 25th June 2013 at 12:21 and at 12:12 (UTC), for which respectively 4 and 5 rebounds have been identified in the seismic traces. In both cases, the subsequent impacts were located within a limited area and could allow the identification of the zones most prone to rockfall impacts. (Fig. 8a, b).

\section{CASE 2: Blocks hurled next to TOR2 station}

Some of the blocks used for the simulations have been hurled from point A in Fig. 2. In this landslide sector, the Maiolica outcrops are characterized by a lower density of fractures. For this reason, we believe that that sector is characterized by higher propagation velocity of the seismic waves. Moreover, the area is characterized by a different morphology, since point A was close to the quarry external limit that acted as a big obstacle, inducing waves reflection (Fig. 10a). Due to the latter reason, the difference between the measured times and the theoretic ones (calculated considering linear distances) is too high to provide a reliable result comparable to the one from 'CASE 1'. It is worth to note that in fact, with the aim to develop a quick and practical method to detect the blocks trajectories, a bi-dimensional grid of the ground has been used, without considering a seismic wave propagation velocity model of the area. As a consequence of the two above mentioned effects, the location procedure failed for the first impacts albeit the user could manually pick the arrival times at each station. Such problem did not affect the following rebounds that matched with the reconstructed path (red line in Fig. 9).

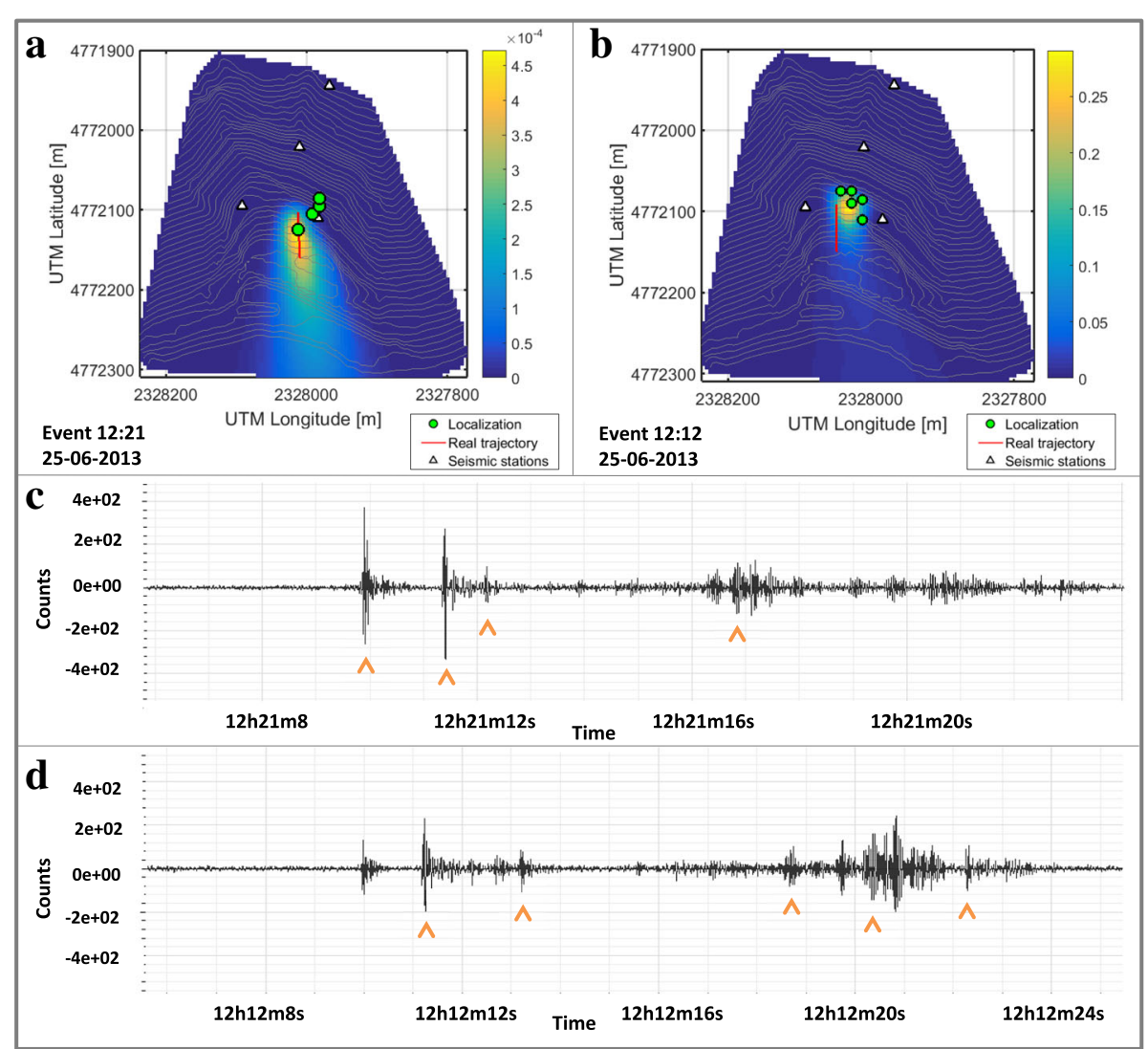

Fig. 8 Localization procedure for CASE 1 events. a Output for the event occurred on 25th June 2013 at 12:21 UTC. b Output for the event occurred on 25th June 2013 at 12:12 UTC. c 12:21 event recorded at TOR4 seismic station. $\mathbf{d}$ 12:12 event recorded at TOR4 seismic station. The orange arrows highlight peaks considered for the elaboration process 


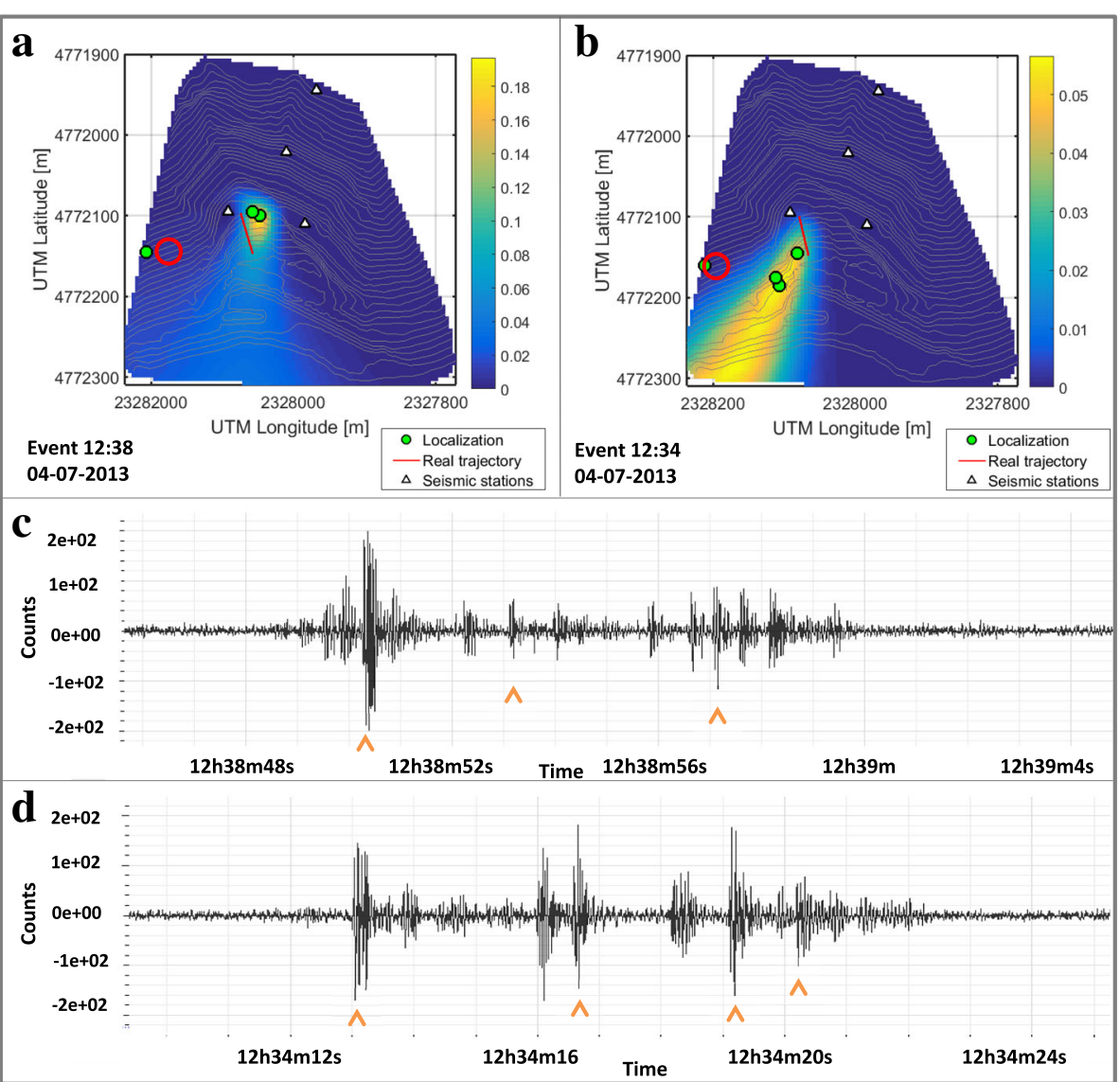

Fig. 9 Localization procedure for CASE2. a Localization output for the event occurred on 4th July at 12:38 UTC. b Localization output for the event occurred on 4th July at 12:34 UTC. Red circles point out the first impact that we were not able to localize correctly. c 12:38 event recorded at TOR4 seismic station. d 12:34 event recorded at TOR4 seismic station. The orange arrows highlight peaks considered for the elaboration process

As proof, we tried to localize the first impact of a block hurled from point $\mathrm{A}$ increasing the propagation waves velocity from $2000 \mathrm{~m} / \mathrm{s}$ to $3000 \mathrm{~m} / \mathrm{s}$. As expected, the obtained result was reliable (Fig. 10b).

\section{CASE 3: Slides on a bedding plane}

Some of the blocks throwed from point B (Fig. 2) slide on a bedding plane. In particular, they fell from a $1 \mathrm{~m}$ high step and then started their glide crossing the bedding plane. In this case seismic traces showed only one meaningful peak due to the first and only impact followed by noise or signals too close in time to be manually picked (Fig. 11b). Moreover, all that was clearly visible after the first impact was recorded by only one or two stations, due to the low energy involved. The single impact was well located, and it matched very well the reconstructed trajectory (Fig. 11a).

\section{Conclusions}

In this paper an algorithm based on the non-linear inversion of seismic waves arrival times recorded by a microseismic network (equipped with 4 stations) to localize the impact points of some boulders artificially thrown along a slope prone to rockfalls has been proposed. Results showed that the methodology is generally reliable and able to retrace the path travelled by the fallen blocks, since the calculated trajectories matched with real ones, unless some errors due to the assumptions. Nevertheless, the applicability of the method to each impact strictly depends on the area of impacts: when the approximation used are respected (bi-dimensional model and homogeneous medium with an isotropic propagation velocity, 'CASE $\left.1^{\prime}\right)$, the identified source point is correctly located, otherwise, if the impact point is located on the outcropping bedrock (near TOR2, 'CASE 2') a modification of the medium velocity value is required in order to obtain a good match between the real and the retraced position.

The accuracy of the methodology is strictly connected to the positioning of the seismic network: the closer the impacts to the instruments, the higher the amplitude of the signals and consequently the manual picking will be feasible at all the stations. 

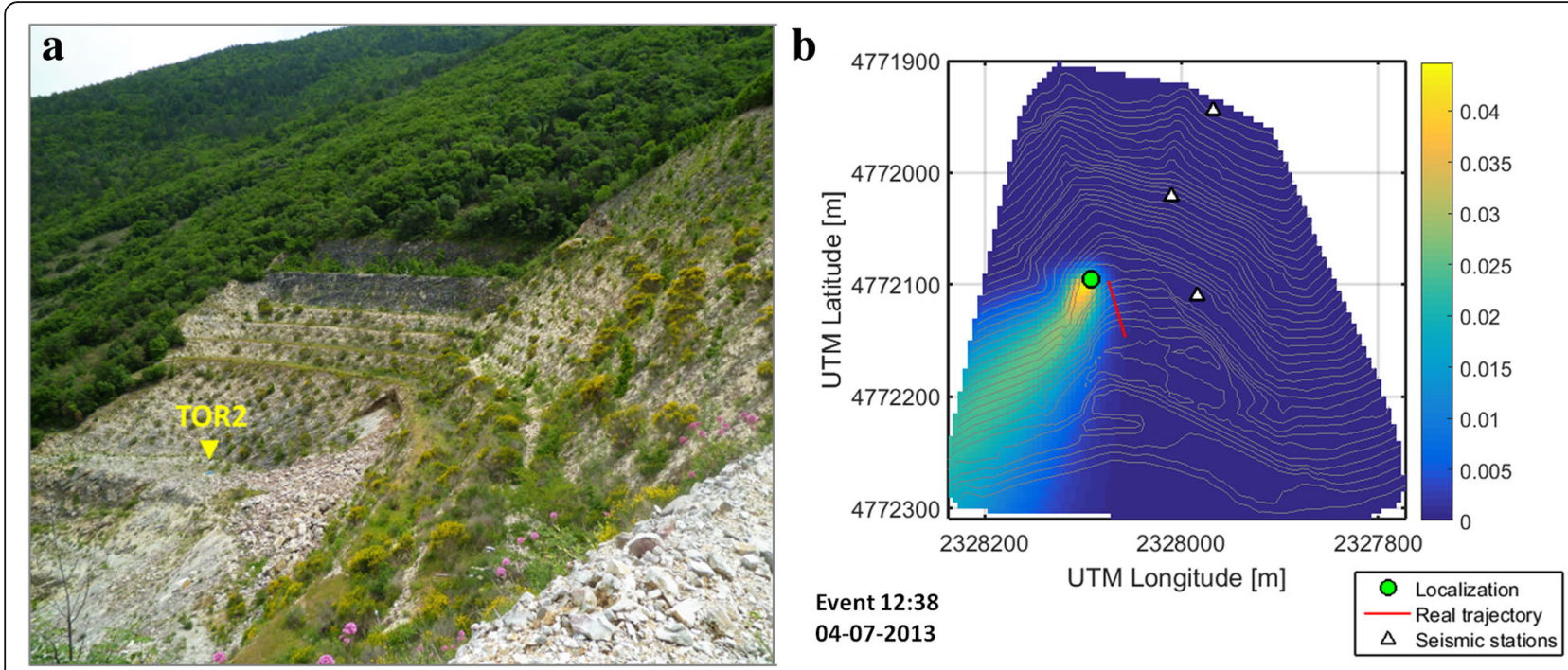

Fig. 10 a Proximity to TOR2 station at the edge of the former quarry. $\mathbf{b}$ Localization of the first impact of the event occurred on 4th July at 12:38 UTC. The localization procedure was made assuming a velocity equal to $3000 \mathrm{~m} / \mathrm{s}$

As far as the blocks size is concerned, no influence on method efficiency has been found, but it's worth to take into account that size of the same order of magnitude have been considered in this work.

The location procedure is intrinsically affected by uncertainties associated with different causes. These are: 1) measuring errors, due to difficulties in detecting the first arrival arising from low SNR or emergent traces; 2) intrinsic instrumental limitations, given by the step sampling of the digital signal $(0,005 \mathrm{~s}) ; 3)$ theoretical approximations, due to the assumption of a constant propagation velocity (assumed equal to $2000 \mathrm{~m} / \mathrm{s}$ ); and

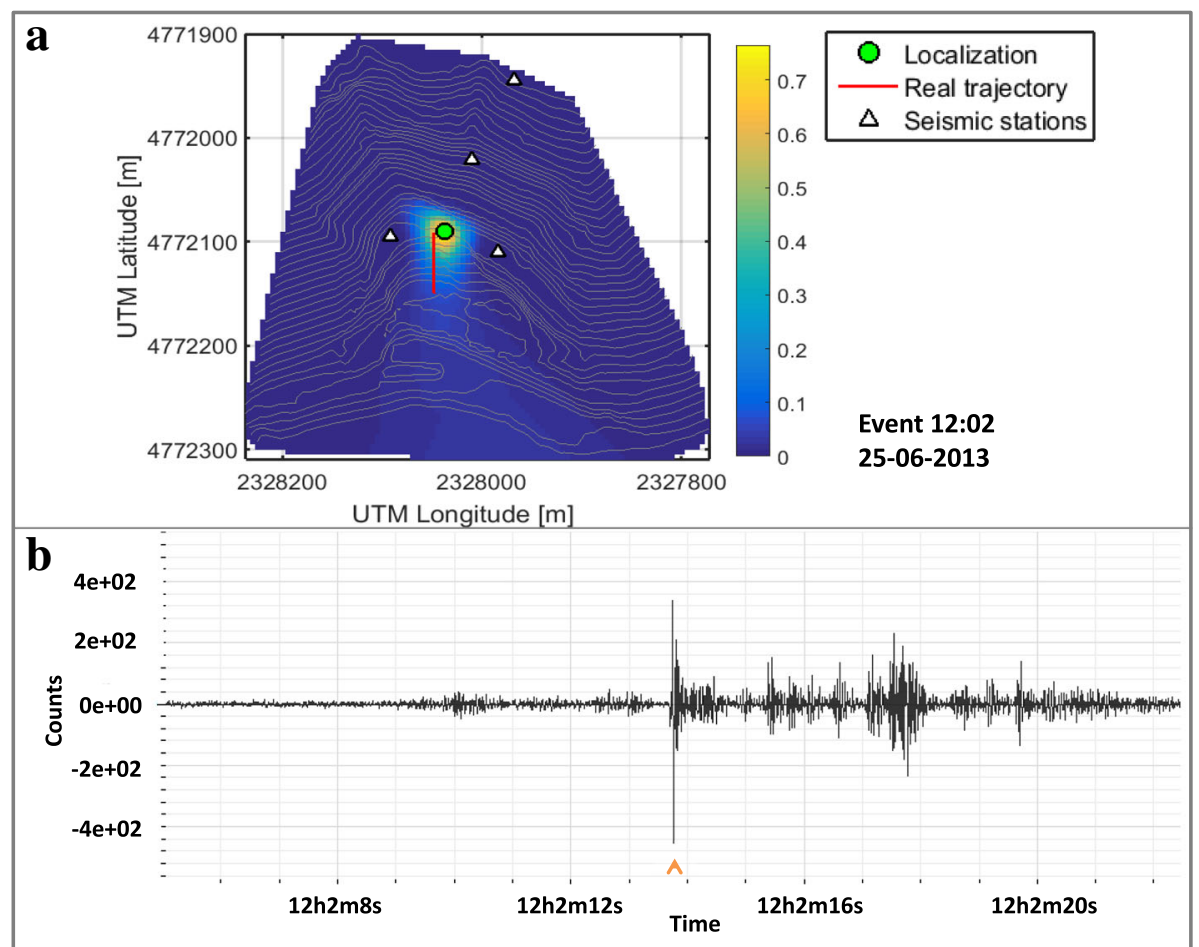

Fig. 11 Localization procedure for CASE 2. a Localization output for the event occurred on 25th June 2013 at 12:02 UTC. b 12:02 event recorded at TOR4 seismic station. The orange arrows highlight peaks considered for the elaboration process 
4) inaccuracies in the ray-tracing procedure, due to the fact that ray trajectories have been calculated as straight lines connecting the nodes of the gridded topographic surface and individual stations. Even under the homogeneous medium approximation, such trajectory cannot be realistic in case of rough topographic surfaces.

The proposed technique provides interesting information about the area that is most prone to impact of the detached blocks, and can represent a useful tool for mapping those areas that need to be protected by defense works (protection works). It is worth noting that the degree of uncertainty could be further reduced by minimizing certain approximations inherent to the method (as example, by using a reliable velocity model).

\section{Acknowledgements}

The work described in this paper was funded by the Italian Ministry of Instruction, University and Research (MIUR) in the framework of the National Research Project PRIN 2009 titled "Integration of monitoring and numerical modeling techniques for early warning of large rockslides". The project was carried out by the Department of Earth Sciences of the University of Florence (National coordinator and responsible for the Research Unit: Prof. Nicola Casagli), the Department of Electrical and Information Engineering "Guglielmo Marconi" of the Università degli Studi di Bologna (responsible for the Research Unit: Prof. Andrea Giorgetti) and the Department of Structural, Building and Geotechnical Engineering of Politecnico di Torino (responsible for the Research Unit: Prof. Marco Barla)

\section{Funding}

Not applicable.

\section{Availability of data and materials}

Not applicable.

\section{Authors' contributions}

TG carried out data collection, conducted analysis and drafted the manuscript. AL AG MB FA helped in draft the manuscript. LL MN FG contributed to the fieldwork and were responsible for the digital modelling. GG and GS gave technical support and conceptual advice and contribute to the preparation of the manuscript. AF and LM handled network maintenance during the whole test period. NC supervised the project. All authors read and approved the final manuscript.

\section{Authors' information}

Not applicable.

\section{Competing interests}

The authors declare that they have no competing interests.

\section{Publisher's Note}

Springer Nature remains neutral with regard to jurisdictional claims in published maps and institutional affiliations.

\section{Author details}

'Department of Earth Sciences, University of Florence, Via Giorgio La Pira 4, 50121 Florence, Italy. ${ }^{2}$ Istituto Nazionale di Geofisica e Vulcanologia, Via U. della Faggiola 32, 56126 Pisa, Italy. ${ }^{3}$ Joint Research Centre of the European Commission, Via Fermi 2147, 21020 Ispra, Italy. ${ }^{4}$ Department of Structural, Building and Geotechnical Engineering, Politecnico di Torino, Corso Duca degli Abruzzi 24, 10129 Torino, Italy. ${ }^{5}$ Department of Electrical, Electronic, and Information Engineering "Guglielmo Marconi", University of Bologna, Viale Risorgimento 2, 40136 Bologna, Italy. ${ }^{6}$ Parsec Foundation (former Prato Ricerche), Via Galcianese 20/H, 59100 Prato, Italy.
Received: 18 October 2017 Accepted: 6 December 2017

Published online: 20 December 2017

\section{References}

Allen, R. 1978. Automatic earthquake recognition and timing from single traces. Bulletin of the Seismological Society of America 68: 1521-1532.

Amorese, D., Font, M., Grasso, J.R., Lotti, A, Saccorotti, G., Ponziani, F. 2015. Applying a change-point detection method on landslide time series: The case of the Torgiovannetto quarry rockslide (central Apennines, Italy). Conference paper: Jag 2015, At Caen, part 2.

Antolini, F. M. Barla, G. Gigli, A. Giorgetti, E. Intrieri, and N. Casagli. 2016. Combined finite-discrete numerical modeling of Runout of the Torgiovannetto di Assisi rockslide in Central Italy. International Journal of Geomechanics 16 (6): 1-16.

Baillifard, F., M. Jaboyedoff, and M. Sartori. 2003. Rockfall hazard mapping along a mountainous road in Switzerland using a GIS-based parameter rating approach. NHESS 3: 435-442.

Barla, M., and F. Antolini. 2016. An integrated methodology for landslides' early warning systems. Landslides 13 (2): 215-228.

Berger, F., C. Quetel, and L.K.A. Dorren. 2002. Forest: A natural protection mean against rockfalls, but with which efficiency? The objectives and methodology of the Rockfor project. International congress INTERPRAEVENT 2002 in the Pacific rim - MATSUMOTO/JAPAN. Congress Publication 2: 815-826.

Bottelin, P., D. Jongmans, D. Daudon, A. Mathy, A. Helmstetter, V. BonillaSierra, H. Cadet, D. Amitrano, V. Richefeu, L. Lorier, L. Baillet, P. Villard, and F. Donzé. 2014. Seismic and mechanical studies of the artificially triggered rockfall at mount Néron (French alps, December 2011). NHESS 14: 3175-3193.

Bour, M., D. Fouissac, P. Dominique, and C. Martin. 1998. On the use of microtremor recordings in seismic microzonation. Soil Dynamics and Earthquake Engineering 17 (7): 465-474

Bruno, F., and F. Martillier. 2000. Test of high-resolution seismic reflection and other geophysical techniques on the Boup landslide in the Swiss alps. Surveys in Geophysics 21 (4): 335-350.

Chen, C., and A.A. Holland. 2016. PhasePApy: A Roboust pure python package for Authomatic identification of seismic phases. Seismological Research Letters 87 (6): 1384-1396

Colombero, C., Comina, C., Vinciguerra, S., Jongmans, D., Baillet, L., Helmstetter, A., Larose, E., Valentin, J. 2016. Passive seismic monitoring of potentially unstable rock masses. Conference: ISRM Eurock 2016, At Cappadocia, Turkey, Volume: Rock Mechanics and Rock Engineering: From the Past to the Future. pp: 1231-1236

Cruden, D.M., and D.J. Varnes. 1996. Landslides types and processes. In Landslide: Investigation and mitigation, National Research Council, 247, Transportation Research Board, ed. A.K. Turner and R.L. Schuster, 36-75.

Dammeier, F., J.R. Moore, F. Haslinger, and S. Loew. 2011. Characterization of alpine rockslides using statistical analysis of seismic signals. Journal of Geophysical Research 116: F04024. https://doi.org/10.1029/2011JF002037.

Dammeier, F., J.R. Moore, C. Hammer, F. Haslinger, and S. Loew. 2016. Automatic detection of alpine rockslides in continuous seismic data using hidden Markov models. Journal of Geophysical Research: Earth Surface 121 (2): 351-371.

Deparis, J., Jongmans, D., Cotton, F., Baillet, L., Thouvenot, F., Hantz, D. 2008. Analysis of Rock-Fall and Rock-Fall Avalanche Seismograms in the French Alps. Bulletin of the Seismological Society of America 98 (4): 1781-1796.

Giorgetti, A., Lucchi, M., Tavelli, E., Barla, M., Gigli, G., Casagli, N., Chiani, M. and Dardari, D. 2016. A Roboust Wireless Sensor Network for Landslide Risk Analysis: System design, Deployment, and Field Testing. IEEE Sensor Journal 16 (16): 6374-6386

Goldstein, P., Snoke, A. 2005. SAC availability for the IRIS Community, Electronic Newsletter, Incorporated Institutions for Seismology, Data Management Center. available at http://ds.iris.edu/ds/newsletter/vol7/no1/sac-availabilityfor-the-iris-community/

Goldstein, P., D. Dodge, M. Firpo, and L. Minner. 2003. In SAC2000: Signal processing and analysis tools for seismologists and engineers. Invited contribution to "the IASPEI international handbook of earthquake and engineering seismology", ed. W.H.K. Lee, H. Kanamori, P.C. Jennings, and C. Kisslinger, 2003. London: Academic Press.

Graziani A., Marsella M., Rotonda T., Tommasi P., Soccodato C. 2009. Study of a rockslide in a limestone formation with clay interbeds. Proc. Int. conf. On rock joints and jointed rock masses, Tucson, Arizona, USA 7th-8th January 2009. 
Günther, A., Hervás, J., Van Den Eeckhaut, M., Reichenbach, P. 2014. Synoptic panEuropean landslide susceptibility assessment: The ELSUS 1000 v1 map. In: Sassa, K., Canuti, P., Yin, Y., (eds) Landslide science for a safer Geoenvironment. https://doi.org/10.1007/978-3-319-04999-1_12.

Halbert, S. E., Buland, R., Hutt, C. R. 1988. Standard for the exchange of earthquake data (SEED), version V2. 0, February 25, 1988. United States geological survey, Albuquerque seismological laboratory, building, 10002.

Helmstetter, A., and S. Garambois. 2010. Seismic monitoring of Séchilienne rockslide (French alps): Analysis of seismic signals and their correlation with rainfalls. Journal of Geophysical Research 115: F03016. https://doi.org/10.1029/ 2009JF001532.

Hibert, C., A. Mangeney, G. Grandjean, C. Baillard, D. Rivet, N.M. Shapiro, C. Satriano, A. Maggi, P. Boisser, V. Ferrazzini, and W. Crawfor. 2014. Automated identification, location, and volume estimation of rockfalls at piton de la Fournaise volcano. Journal of Geophysical Research - Earth Surface 119: 1082-1105. https://doi.org/10.1002/2013JF002970.

Intrieri, E., G. Gigli, F. Mugnai, R. Fanti, and N. Casagli. 2012. Design and implementation of a landslide early-warning system. Engineering Geology 147-148: 124-136.

Jongmans, D., and S. Garambois. 2007. Geophysical investigation of landslides: A review. Bulletin de la Société Géologique de France. 178 (2): 101-112.

Lacroix, P., and A. Helmstetter. 2011. Location of seismic signals associated with microearthquakes and rockfalls on the Séchilienne landslide, French alps. Bulletin of Seismological Society of America 101 (1): 341-353.

Levy, C., D. Jongmans, and L. Baillet. 2011. Geophysical Journal International 186: 293-310.

Lotti, A., G. Saccorotti, A. Fiaschi, L. Matassoni, G. Gigli, V. Pazzi, and N. Casagli. 2015. Seismic monitoring of a rockslide: The Torgiovannetto quarry (central Apennines, Italy). Engineering Geology for Society and Territory 2: 1537-1540.

Manconi, A., M. Picozzi, V. Coviello, F. De Santis, and L. Elia. 2016. Real-time detection, location, and characterization of rockslides using broadband regional seismic networks. Geophysical Research Letters 43 (13): 6960-6967.

McCann, D.M., and A. Forster. 1990. Reconnaissance geophysical methods in landslide investigations. Engineering Geology 29 (1): 59-78.

Moser, T.J., T. Van Eck, and G. Nolet. 1992. Hypocenter determination in strongly heterogeneous earth models using the shortest path method. Journal of Geophysical Research 97: 6563-6572.

Norris, R.D. 1994. Seismicity of rockfalls and avalanches at three Cascade range volcanoes: Implications for seismic detection of hazardous mass movements. Bulletin of the Seismological Society of America 84 (6): 1925-1939.

Ponziani, F., N. Berni, C. Pandolfo, M. Stelluti, L. Broca, and L. Moramarco. 2011. An integrated approach for an operative early-warning system of landslides forecasting based on rainfall thresholds and soil moisture assessment. Vienna-Austria: European Geosciences Union, general assembly 3 April 2011

Rocca, F. 1983. Prontuario del Geofisico - Seconda parte. Documento Agip, APVE, GEOF86

Saccorotti, G., B. Chouet, M. Martini, and R. Scarpa. 1998. Bayesian statistics applied to the location of the source of explosions at Stromboli Volcano, Italy. Bulletin of the Seismological Society of America 88 (5): 1099-1111.

Schuster, R.L., and Leighton, R.W. 1988. Socioeconomic significance of landslides and mudflows. In: Kozlovskii, E.A. (ed.) Landslides and Mudflows. UNESCO/ UNDP, moscow, pp. 131-141.

Scott, J.B., T. Rasmussen, B. Luke, W.J. Taylor, J.L. Wagoner, S.B. Smith, and J.N. Louie. 2006. Shallow shear velocity and seismic microzonation of the urban Las Vegas, Nevada, basin. Bulletin of the Seismological Society of America 96 (3): 1068-1077.

Suriñach, E., I. Vilajosana, G. Khazaradze, B. Biescas, G. Furdada, et al. 2005. Seismic detection and characterization of landslides and other mass movements. Natural Hazards and Earth System Science, Copernicus Publications on behalf of the European Geosciences Union 5 (6): 791-798.

Tarantola, A., and B. Valette. 1982. Generalized nonlinear inverse problems solved using the least squares criterion. Reviews of Geophysics 20 (2): 219-232.

Vilajosana, I., E. Suriñach, A. Abellán, G. Khazaradze, D. Garcia, and J. Llosa. 2008. Rockfall induced seismic signals: Case study in Montserrat, Catalonia. NHESS 8: $805-812$

\section{Submit your manuscript to a SpringerOpen ${ }^{\circ}$ journal and benefit from:}

- Convenient online submission

- Rigorous peer review

- Open access: articles freely available online

- High visibility within the field

Retaining the copyright to your article

Submit your next manuscript at $>$ springeropen.com 